\title{
Photoluminescence and Electron Paramagnetic Resonance Studies of Bulk GaN Doped with Gadolinium
}

\author{
Z. LiPIŃSKA ${ }^{a}$, M. PAWŁOWSKI $^{b}$, H. ŻOŁnIEROWICZ ${ }^{a}$, \\ A. Wysmolek ${ }^{a, *}$, M. PAlczewska ${ }^{b}$, M. KAmińska $^{a}$, \\ A. Twardowski ${ }^{a}$, M. BoćKowski ${ }^{c}$ AND I. GrzegorY ${ }^{c}$ \\ ${ }^{a}$ Institute of Experimental Physics, Warsaw University \\ Hoża 69, 00-681 Warsaw, Poland \\ ${ }^{b}$ Institute of Electronic Materials Technology \\ Wólczyńska 133, 01-919 Warsaw, Poland \\ ${ }^{c}$ Institute of High Pressure Physics, Polish Academy of Sciences \\ Sokołowska 29, 01-142 Warsaw, Poland
}

\begin{abstract}
Photoluminescence and electron paramagnetic resonance experiments on strain free GaN bulk crystals of wurtzite structure doped with gadolinium are reported. Efficient gettering of residual GaN donors by Gd was observed. Electron paramagnetic resonance showed that Gd ion incorporated into GaN lattice had $\mathrm{Gd}^{3+}\left(4 f^{7}\right)$ configuration. The observed photoluminescence spectra were explained as due to intracenter $\mathrm{Gd}^{3+}\left(4 f^{7}\right)$ transitions. No ferromagnetic behavior was detected.
\end{abstract}

PACS numbers: 78.55.Cr, 71.55.-i, 71.35.--y

\section{Introduction}

Theoretical predictions of possible room temperature ferromagnetism in GaN diluted with manganese strongly motivated studies of nitride based diluted magnetic semiconductors (DMS) with transition metals or rare-earths. Over the last years, above room temperature ferromagnetism for MBE grown GaN layers weakly doped with Gd has been reported by different researchers [1]. The estimated average value of magnetic moment per Gd ion has been obtained as high as 500 times that of the Gd atomic value. It has been argued that the observed enhancement

*corresponding author; e-mail: Andrzej Wysmolek@fuw.edu.pl 
of the average magnetic moment resulted from strong contribution of GaN host lattice to macroscopic magnetization of the whole material [1]. The origin of such behavior remains still puzzling and more studies of GaN:Gd are required to clarify this issue.

In this paper we present photoluminescence (PL) and electron paramagnetic resonance (EPR) experiments on strain free GaN bulk crystals of wurtzite structure doped with gadolinium. Both PL and EPR techniques are well known as powerful experimental tools to study electronic and magnetic properties of transition metals and rare-earth in semiconductors, and they have not been exploited up to now for GaN:Gd system.

\section{Samples and experiment}

Strain free GaN bulk crystals of wurtzite structure doped with Gd were grown at High Pressure Research Center, from the solution of nitrogen in liquid gallium under high $\mathrm{N}_{2}$ pressure ( $\mathrm{HP}$ ) of $1.5 \mathrm{GPa}$ and at elevated temperatures of about $1500^{\circ} \mathrm{C}$. Doping with Gd was obtained by adding this element to the solution. Several samples with different nominal Gd concentrations were studied.

EPR experiments were carried out using a Brucker ESP-300 X-band spectrometer with $100 \mathrm{kHz}$ field modulation and phase-sensitive detection, which operated at a microwave frequency of about $9.4 \mathrm{GHz}$. A helium gas-flow Oxford Instruments cryostat was used for variable temperature measurements, starting from $6 \mathrm{~K}$. PL experiments were performed at $4.2 \mathrm{~K}$ using continuous-flow cryostat. PL was excited by means of $3.813 \mathrm{eV}$ line of $\mathrm{He}-\mathrm{Cd}$ laser. The exciting light was focused on the sample using microscope objective, providing a spot size of a few microns. The excitation density resulting on the sample was estimated to be in the range of $10^{4} \mathrm{~W} / \mathrm{cm}^{2}$. The spectra were analyzed with a single $0.5 \mathrm{~m}$ monochromator, equipped with a UV-enhanced CCD camera. The spectral resolution provided by the experimental setup was better than $0.2 \mathrm{meV}$.

\section{Results and discussion}

Representative for the investigated samples EPR spectrum, obtained for magnetic field perpendicular to the $c$ GaN axis and measured at $T=10 \mathrm{~K}$ is shown in Fig. 1. This spectrum did not changed much for room temperatures scan. It is characteristic of $\mathrm{Gd}^{3+}\left(4 f^{7}\right)$ configuration. Although such spectrum has not been observed for GaN:Gd before, it is very much alike the seven fine structure lines reported for $\mathrm{Gd}^{3+}\left(4 f^{7}\right)$ in single wurtzite $\mathrm{ZnO}$ crystals [2]. The free $\mathrm{Gd}^{3+}\left(4 f^{7}\right)$ ion has an ${ }^{8} S_{7 / 2}$ ground state. As the crystal field is always weak for rare earth ions, this remains the ground state in a crystal too. GaN has a hexagonal crystal structure of the wurtzite type with a sixfold symmetry around the polar $c$ axis. The general Hamiltonian for $\mathrm{Gd}^{3+}$ impurity on a cation site can be written as $[3,4]$ :

$$
H=g \beta \boldsymbol{H} \boldsymbol{S}+B_{2}^{0} O_{2}^{0}+B_{4}^{0} O_{4}^{0}+B_{4}^{3} O_{4}^{3}+B_{6}^{0} O_{6}^{0}+B_{6}^{3} O_{6}^{3}+B_{6}^{6} O_{6}^{6} .
$$




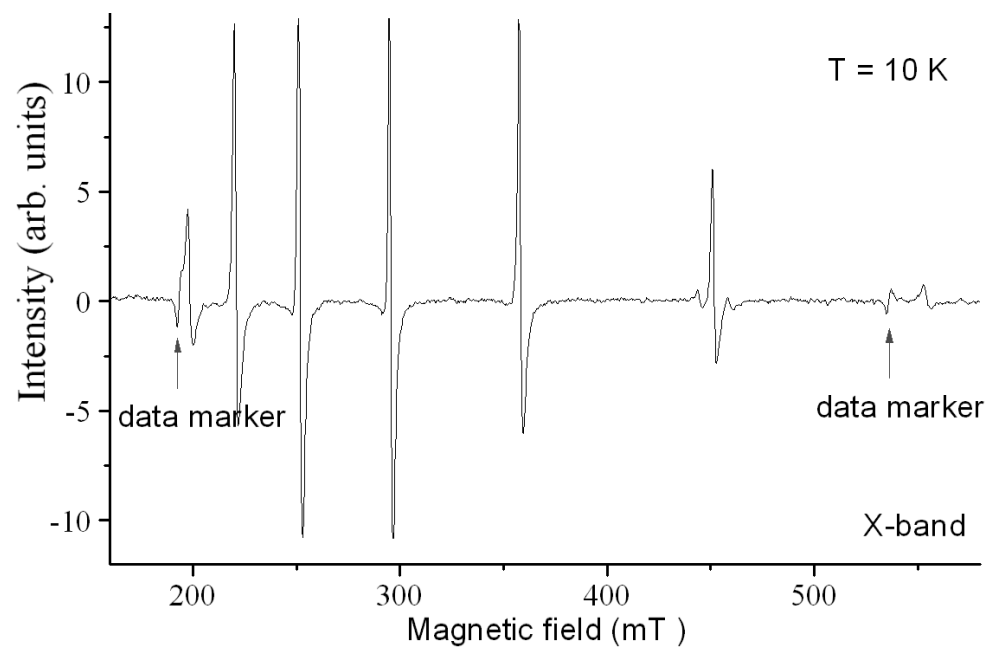

Fig. 1. GaN: $\mathrm{Gd}^{3+}\left(4 f^{7}\right)$ spectrum for $\boldsymbol{H} \perp \boldsymbol{c}$.

The constants $B_{m}^{\mathrm{n}}$ are empirical parameters to be determined from the experimental results and $O_{m}^{\mathrm{n}}$ are Steven's operators. The spin Hamiltonian contains six crystal field parameters and the evaluation of all these is a difficult task. However, when $\boldsymbol{H} \| \boldsymbol{z}$, transitions corresponding to $\Delta M= \pm 1$ can be calculated to the second order perturbation and one can neglect the contribution from the terms with $B_{4}^{3}, B_{6}^{3}$, and $B_{6}^{6}$. This way we obtained $g_{\|}=1.968 \pm 0.002$. In the case of $\boldsymbol{H}$ not parallel or perpendicular to the $c$ axis, the spectrum became rather complex and it was possible to observe a splitting of all the lines, due to the fact that two $\mathrm{Gd}^{3+}$ sites in the unit cell of the wurtzite lattice were magnetically distinguishable. If the condition $g \beta H \gg B_{2}^{0}, B_{4}^{0}, B_{6}^{0}$ is fulfilled, the angular dependence of $\mathrm{Gd}^{3+}\left(4 f^{7}\right)$ resonances can be calculated $[2,3]$. However, we still need to check whether this is our case and therefore the work with the aim of determining these $B_{m}^{\mathrm{n}}$ parameters is under progress.

The observed Dysonian shape of the EPR lines originated from the presence of high concentration of free electrons in the measured GaN:Gd samples. However, comparison with undoped HP bulk GaN crystals indicated that the value of free electron concentration was definitively lower in Gd doped crystals. This indicated a purification role of Gd from unintentional donors (presumably oxygen). A similar efficient gettering of residual donors by rare earths has been observed for other crystals as well [5].

A typical low temperature photoluminescence spectrum of GaN:Gd in a wide energy range is presented in Fig. 2. It consists of characteristic of GaN broad band centered around $2.3 \mathrm{eV}$, frequently referred as yellow luminescence, and step-like structure above band-gap of GaN, which is characteristic of heavily $n$-type doped material with free electron concentration higher than $10^{18} \mathrm{~cm}^{-3}$. However, free electron concentration in typical undoped $\mathrm{HP}$ GaN crystals is higher than that, 


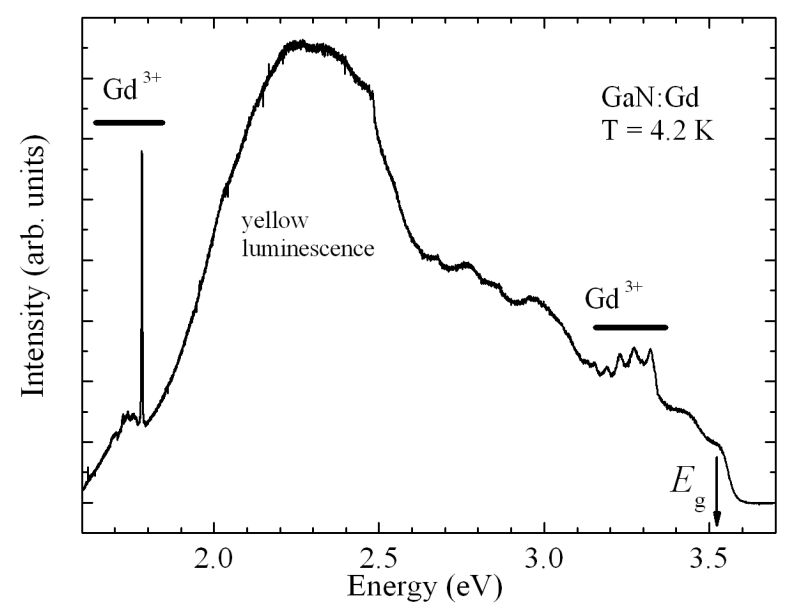

Fig. 2. The photoluminescence spectrum of a bulk $n$-type GaN sample doped with Gd, measured at temperature of $4.2 \mathrm{~K}$.

about $5 \times 10^{19} \mathrm{~cm}^{-3}$, and very weak luminescence in band gap range is observed. This observation is one more indication of efficient gettering role of Gd.

New features in PL spectrum were observed in two energy ranges: close to GaN band gap (3.1-3.6 eV) and in the "red" range of 1.6-1.8 eV (see Figs. 3 and 4).

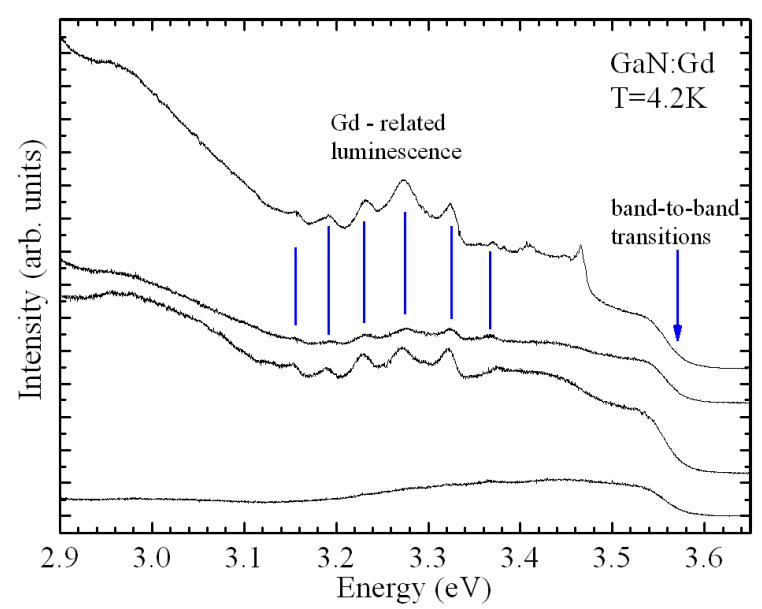

Fig. 3. High energy part of the emission measured for different spots on the sample. The Gd-related emission consists of six well-resolved lines separated by about 35-42 meV.

Up to now, in AlN and other wide gap materials doped with Gd, PL has been observed at about $3.95 \mathrm{eV}$ and identified as originating from transition from first $\mathrm{Gd}^{3+}\left(4 f^{7}\right)$ excited level ${ }^{6} P_{7 / 2}$ to its ground state ${ }^{8} S_{7 / 2}[6-8]$. There is some 


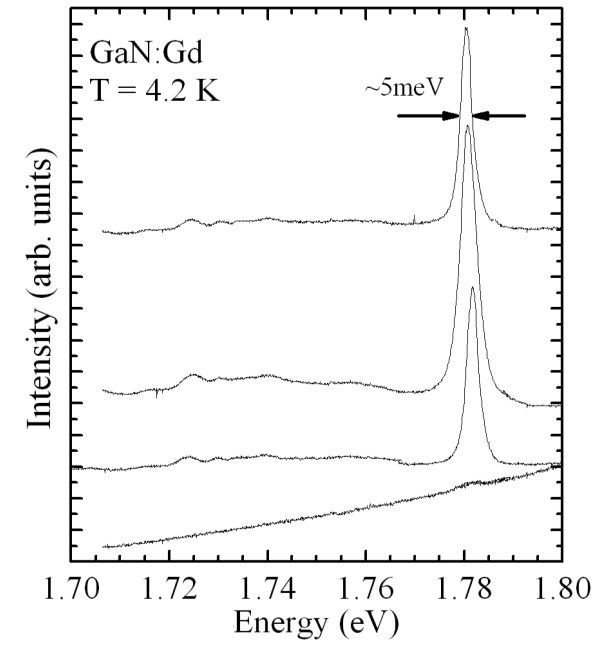

Fig. 4. Low energy part of the emission measured for different spots on the GaN:Gd sample.

possibility that the structure observed by us at $3.3 \mathrm{eV}$ is also due to this specific transition. GaN is less ionic compound than AlN, so one can expect a bigger influence of crystal field for GaN and, as a consequence, reduction of energy distance between Gd levels. However, the shift by as much as $0.65 \mathrm{eV}$ is rather doubtful, and also the lines observed by us are much broader that the sharp peaks characteristic of rare earth internal transitions. Further studies involving PL measurements in magnetic field are needed in order to clarify this issue.

In the "red" range, sharp structure with main line around $1.78 \mathrm{eV}$ energy was detected. The FWHM of this line was as small as $5 \mathrm{meV}$. We would like to relate this structure to $\mathrm{Gd}^{3+}\left(4 f^{7}\right)$ internal transition. The most probable seemed to be transitions between excited states, from ${ }^{6} G_{7 / 2}$ to ${ }^{6} P_{J}(J=7 / 2,5 / 2,3 / 2)$ states. Similar transitions have been observed for Gd doped $\mathrm{LiYF}_{4}$ crystals at about $2 \mathrm{eV}$, and it has been shown that this is a very efficient channel for radiative transitions.

Judging from EPR and PL results, Gd dopant was incorporated to the studied samples. However, no ferromagnetic behavior of the samples was observed by means of EPR. This creates some doubts whether ferromagnetism observed by others was really related to GaGdN system.

\section{Conclusions}

Efficient purification of HP GaN crystals by Gd was observed. EPR and PL experiments showed that gadolinium incorporates as $\mathrm{Gd}^{3+}\left(4 f^{7}\right)$. No ferromagnetism due to this impurity was detected. 


\section{References}

[1] S. Dhar, O. Brandt, M. Ramsteiner, V.F. Sapega, K.H. Ploog, Phys. Rev. Lett. 94, 037205 (2005).

[2] A. Hausmann, Solid State Commun. 7, 579 (1969).

[3] A. Abragam, B. Bleaney, Electron Paramagnetic Resonance of Transition Ions, Clarendon Press, Oxford 1970, p. 335.

[4] J.W. Orton, Electron Paramagnetic Resonance, Ilivffe Books LTD, London 1968, p. 216.

[5] A. Stapor, J. Raczyńska, H. Przybylińska, A. Sienkiewicz, K. Fronc, J.M. Langer, Mater. Sci. Forum 10-12, 633 (1986).

[6] U. Vetter, J. Zenneck, H. Hofsass, Appl. Phys. Lett. 83, 2145 (2003).

[7] R.T. Wegh, H. Donker, A. Meijerink, Phys. Rev. B 56, 13841 (1997).

[8] J. Sytsma, G.F. Imbusch, G. Blasse, J. Chem. Phys. 91, 1456 (1989). 